\title{
Precise pathologic diagnosis and individualized treatment improve the outcomes of invasive micropapillary carcinoma of the breast: a 12-year prospective clinical study
}

\author{
Weidong $\mathrm{Li}^{1,2} \cdot$ Yunwei Han ${ }^{1,2} \cdot$ Chijuan Wang ${ }^{1,2} \cdot$ Xiaojing Guo ${ }^{1,2} \cdot$ Beibei Shen ${ }^{1,2} \cdot$ Fangfang Liu ${ }^{1,2}$. \\ Chengying Jiang ${ }^{1,2} \cdot$ Yaqing $\mathrm{Li}^{1,2} \cdot$ Yiling Yang ${ }^{1,2} \cdot$ Ronggang Lang $^{1,2} \cdot \mathrm{Yu} \mathrm{Fan}^{1,2} \cdot \mathrm{Feng} \mathrm{Gu}^{1,2} \cdot \mathrm{Yun} \mathrm{Niu}^{1,2}$. \\ Xinmin Zhang $^{3} \cdot$ Lanjing Zhang ${ }^{4,5,6,7} \cdot$ Hannah Y Wen ${ }^{8} \cdot$ Li Fu $^{1,2}$
}

Received: 11 September 2017 / Revised: 28 December 2017 / Accepted: 30 December 2017 / Published online: 5 February 2018

(c) United States \& Canadian Academy of Pathology 2018

\begin{abstract}
Invasive micropapillary carcinoma of the breast is a histologic subtype of breast cancer and associated with high incidence of lymphovascular invasion, lymph node metastasis and poor prognosis. The aim of this prospective study was to investigate the impact of precise pathologic diagnosis and individualized treatment on the outcomes of invasive micropapillary carcinoma of the breast. The study group included 2299 women with invasive micropapillary carcinoma diagnosed at Tianjin Medical University Cancer Institute and Hospital between January 2004 and December 2015. In the study group, specimens were examined with the method of whole-specimen orientation and serial sectioning, and patients received precise pathological diagnosis and individualized treatment. The control group of invasive micropapillary carcinoma consisted of 163 cases, identified through a retrospectively review of 9056 invasive carcinomas diagnosed at our institution between January 1989 and December 2003 using the standard pathology-evaluation method (i.e., not using the wholespecimen orientation and serial-sectioning method). The clinicopathological features, treatments and outcomes were compared between the two groups. The incidence of invasive micropapillary carcinoma in the study group was 6\% (2299/ 39,714 cases), significantly higher than that of the control group (2\%; 163/9056 cases). The 5-year disease-free survival in

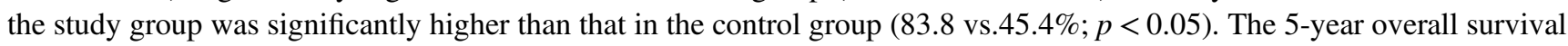
was significantly increased from $57.4 \%$ in the control group to $90.9 \%$ in the study group $(p<0.05)$. In the multivariate analysis, lymphovascular invasion, estrogen receptor status and lymph node metastasis were independent prognostic factors. Although invasive micropapillary carcinoma of the breast is associated with poor prognosis, precise pathologic diagnosis and individualized treatment improved the disease-free survival and overall survival of invasive micropapillary carcinoma patients. Precise pathological diagnosis is the premises for individualized treatments and for improving the outcomes of patients with invasive micropapillary carcinoma of the breast.
\end{abstract}

Weidong Li and Yunwei Han contributed equally to this work.

Electronic supplementary material The online version of this article (https://doi.org/10.1038/s41379-018-0024-8) contains supplementary material, which is available to authorized users.

Hannah Y Wen

weny@mskcc.org

$\bowtie \mathrm{Li} \mathrm{Fu}$

fulijyb@hotmail.com

fuli@tmu.edu.cn

Extended author information available on the last page of the article

\section{Introduction}

Invasive micropapillary carcinoma of the breast is a rare histologic subtype, first described by Fisher et al. [1] in 1980. The term "invasive micropapillary carcinoma" of the breast was first proposed by Siriaunkgul and Tavassolil [2] in 1993. This micropapillary growth pattern was subsequently identified in tumors of other anatomic sites, such as urinary bladder [3], colon [4], and lung [5], and was found to be associated with aggressive biologic behaviors and poor prognoses.

Invasive micropapillary carcinoma is a morphologically distinct form of breast cancer, composed of small, hollow or morula-like clusters of cancer cells, surrounded by clear 
stromal spaces $[1,2]$. The tumor cells display an "insideout" arrangement with the luminal aspect of the cell present on outer surface of the cluster [6,7]. Invasive micropapillary carcinoma is a relatively rare subtype of invasive breast cancer, which accounts for $<2 \%$ to $\sim 8 \%$ of all breast cancers [8-12]. Invasive micropapillary carcinoma is wellknown for its high incidence of lymphovascular invasion, lymph node metastasis, and poor prognosis [13-16]. Despite the association with an unfavorable outcome, the current standard treatment for invasive micropapillary carcinoma is the same as that for invasive ductal carcinoma of no special type.

Invasive micropapillary carcinoma is listed as a special histologic subtype of breast cancer in the World Health Organization (WHO) Classification of Tumors of the Breast in the 2003 and 2012 editions [17, 18]. Our group carried out a series of studies on invasive micropapillary carcinoma, including clinical pathological characteristics [13], biological features [19-24], histopathologic criteria for diagnosis, prognosis, and mechanisms of metastasis [25-27]. We previously reported [6] the outcomes of 100 breast carcinomas with an invasive micropapillary carcinoma component, diagnosed at our institution between 1989 and 2001. Our data demonstrated that, even if invasive micropapillary carcinoma is present as a minor component and mixed with non-micropapillary invasive carcinoma, there is still higher incidence of lymphovascular invasion, lymph node metastasis, and recurrence. We therefore recommended that the invasive micropapillary carcinoma component, even presented as a small proportion, should be recognized and indicated in the pathology report $[6,13]$. The percentage of the invasive micropapillary carcinoma component, the extent of lymphovascular invasion, and the receptor status of each histological type should be described in detail in order to guide individualized clinical treatment $[6,13]$. We have developed the method of whole-specimen orientation and serial sectioning for three-dimensional specimen imaging, and documentation of the site of all lesions in mastectomy specimens, referred as precise pathologic diagnosis.

Here we report a 12-year prospective study on the 2299 cases of invasive micropapillary carcinoma of the breast with precise pathological diagnosis and individualized treatment, and compared these cases with the 163 retrospective-identified cases of the invasive micropapillary carcinoma diagnosed by routine pathological diagnosis (including 100 cases of breast invasive micropapillary carcinoma previously reported [6]) without individualized treatment. Our results indicate that precise pathologic diagnosis and individualized treatment improve the outcomes of invasive micropapillary carcinoma patients.

\section{Material and methods}

\section{Patient cohort}

The study cohort included all breast carcinomas containing invasive micropapillary carcinoma component diagnosed from January 2004 to December 2015 at Tianjin Medical University Cancer Institute and Hospital in China. All mastectomy specimens were examined using the method of whole-specimen orientation and serial sectioning [13, 28]. In brief, the specimens were serially sectioned at $5 \mathrm{~mm}$ intervals and thoroughly examined. Photographs were taken to annotate the specimen. All non-fatty breast tissue and tumor were fixed in formalin and embedded in paraffin, followed by hematoxylin and eosin staining for microscopic examination. All tumors were entirely submitted for pathologic diagnosis.

We also reviewed the breast carcinomas diagnosed without using the method of whole-specimen orientation and serial sectioning from January 1989 to December 2003, and identified the cases with an invasive micropapillary carcinoma component, which would serve as the control group.

\section{Pathological diagnosis}

Four senior breast pathologists (L Fu, Y Niu, Y Fan and RG Lang) reviewed the cases and confirmed the diagnose of breast cancer with invasive micropapillary carcinoma component according to the WHO classification of breast tumors $[17,18]$. The proportion of the invasive micropapillary carcinoma component, histologic grade, lymphovascular invasion, and lymph node status were recorded. The invasive micropapillary carcinoma percentage was classified into $\leq 25 \%$ (Fig. 1a), 26-50\%, 51-75\%, and $>75 \%$ (Fig. 1b).

\section{Immunohistochemical staining}

Immunohistochemical analyses were performed on $4-\mu \mathrm{m}$ sections of formalin-fixed, paraffin-embedded samples. Positive control sections were included in each batch of staining, while the staining with normal mouse serum was used as the negative control.

Tumors were stained with epithelial membrane antigen (clone E29, DAKO, Denmark) and polymorphic epithelial mucin (clone EPR1023, Abcam, UK) to identify the "insideout" arrangement, in order to confirm the diagnosis of invasive micropapillary carcinoma. Epithelial membrane antigen (Fig. 1c) and polymorphic epithelial mucin (Fig. 1d) were expressed in the cell membrane and display the reversed polarity. Estrogen receptor (clone SP1, Zymed), progesterone receptor (clone SP2, Zymed) and human epidermal growth factor receptor 2 (DAKO HercepTest TM, 

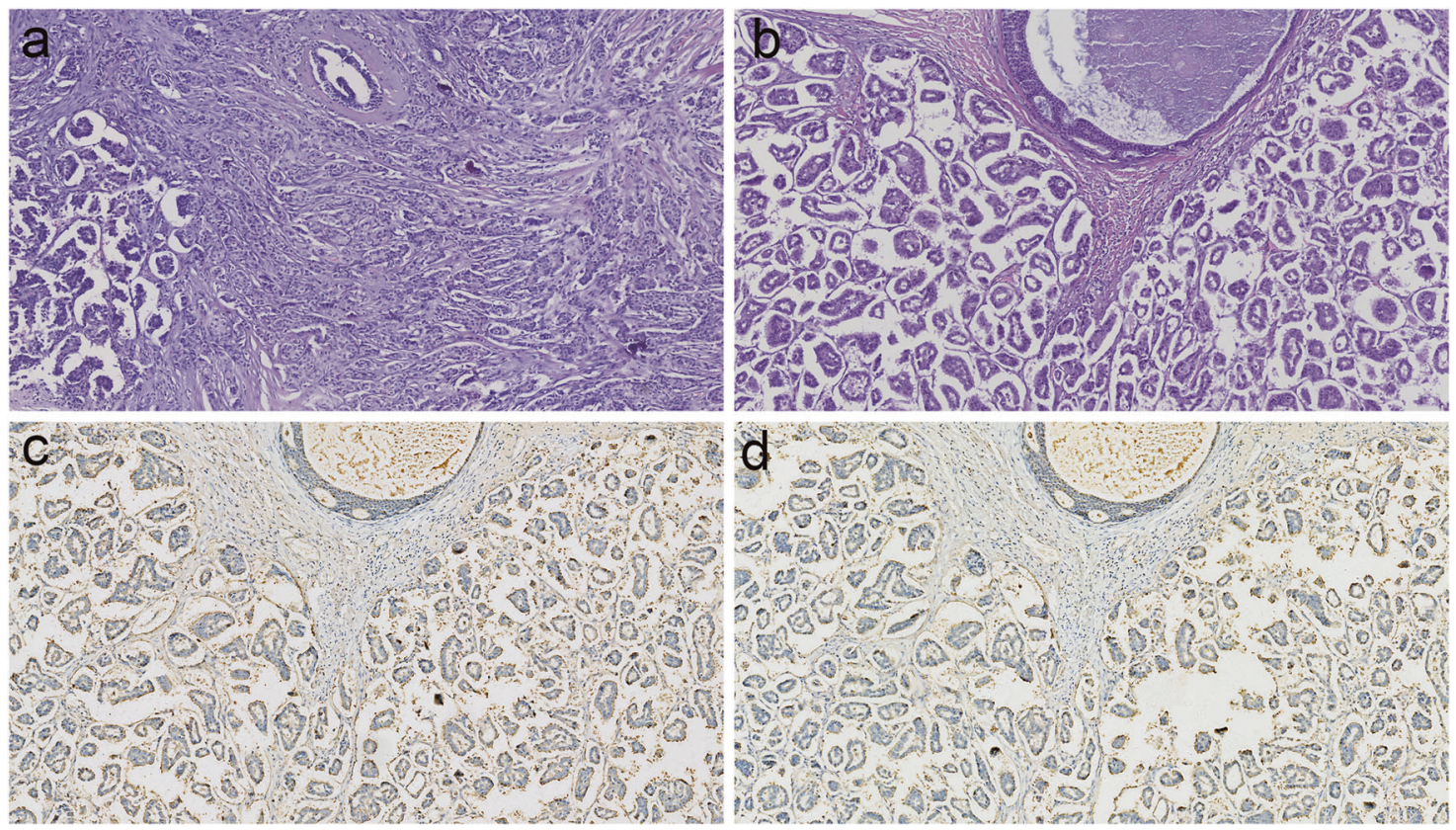

Fig. $1 \mathrm{H} \&$ E stains of invasive breast carcinoma with <25\% micropapillary component (Fig. 1a), with $>75 \%$ micropapillary component (Fig. 1b), and immunohistochemical stains for epithelial membrane antigen (Fig. 1c) and polymorphic epithelial mucin (Fig. 1d)

Denmark) were assessed according to the American Society of Clinical Oncology /College of American Pathologists guidelines [29, 30].

\section{Statistically analyze}

The clinic-pathologic characteristics were compared between the study and control groups using $X^{2}$ test, the Mann-Whitney $U$ test, and the Kruskal-Wallis test. Univariate and multivariate logistic regression models were applied to analyze the predictors for disease-free survivals, and overall survivals. Disease-free and overall survivals were calculated from the date of surgery to the date of locoregional recurrence and or distant metastasis, and death, respectively. The date of the last follow-up or visit was used to calculate survival for patients without recurrence. The curves of disease-free survival and overall survival were calculated using the Kaplan-Meier method, and the differences between the curves were evaluated by means of the log rank test. $P<0.05$ was considered statistically significant. SPSS 22.0 software for Windows (IBM, NY, USA) was used for the analyses.

\section{Results}

\section{Clinicopathological characteristics}

The study group of invasive micropapillary carcinoma consisted of 2299 cases, out of a total of 39714 invasive breast carcinomas diagnosed in the study period. A total of 163 invasive micropapillary carcinomas were identified through the retrospective review (control group), out of 9056 invasive breast carcinomas diagnosed prior to the study period. The clinicopathologic features of 2299 cases of study group invasive micropapillary carcinoma and 163 cases of control group invasive micropapillary carcinoma are summarized in Table 1. The diagnostic rate of invasive micropapillary carcinoma was higher in the study group than that in the control group (6 vs. $2 \%, p<0.05$ ). In addition, the rate of invasive micropapillary carcinoma was increased annually in our hospital, from 3 in 2004 to $7 \%$ in 2015.

In the study group, invasive micropapillary carcinoma constituted $\leq 25 \%$ of tumor in $1148(50 \%)$ cases, $26-50 \%$ in $560(25 \%)$ cases, $51-75 \%$ in $156(7 \%)$ cases, and $>75 \%$ in $412(18 \%)$ cases. In the control group, invasive micropapillary carcinoma constituted $\leq 25 \%$ in $32(20 \%)$ cases, $26-50 \%$ in $39(24 \%)$ cases, $51-75 \%$ in $38(23 \%)$ cases, and $>75 \%$ in $54(33 \%)$ cases. Diagnostic rate of invasive micropapillary carcinoma was increased significantly in the study group, especially in the subgroup with $\leq 25 \%$ invasive micropapillary carcinoma component $(p<0.05)$.

Compared with the control group, the study group had a smaller tumor size $(p=0.012)$, and less-aggressive features including lower incidence of lymphovascular invasion (45 vs. $53 \% ; p=0.030)$ and lymph node metastasis (68 vs. $84 \% ; p<0.01)$, fewer lymph node metastasis per case $(6.5$ vs. $12.4 ; p<0.01)$, higher rates of positive estrogen- 
Table 1 Clinicopathological characteristics of the control group and study group invasive micropapillary carcinoma

\begin{tabular}{|c|c|c|c|}
\hline Characteristics & $\begin{array}{l}\text { CgIMPC }(n= \\
163)(n, \%)\end{array}$ & $\begin{array}{l}\text { SgIMPC }(n= \\
\text { 2299) }(n, \%)\end{array}$ & $P$ value \\
\hline Age at diagnosis (years) & & & $<0.001$ \\
\hline$\leq 50$ & $94(58)$ & $969(42)$ & \\
\hline$>50$ & $69(42)$ & $1330(58)$ & \\
\hline Tumor size (mm) & & & 0.012 \\
\hline$\leq 20$ & $26(17)$ & $591(37)$ & \\
\hline $20-50$ & $102(68)$ & $1385(62)$ & \\
\hline$>50$ & $22(15)$ & $255(11)$ & \\
\hline IMPC proportion & & & $<0.001$ \\
\hline$\leq 25 \%$ & $32(20)$ & $1148(50)$ & \\
\hline $26-50 \%$ & $39(24)$ & $560(25)$ & \\
\hline $51-75 \%$ & $38(23)$ & $156(7)$ & \\
\hline$>75 \%$ & $54(33)$ & $412(18)$ & \\
\hline No of LNM & & & $<0.01$ \\
\hline 0 & $26(16)$ & $722(32)$ & \\
\hline $1-3$ & $30(19)$ & $563(25)$ & \\
\hline$\geq 4$ & $105(65)$ & $982(43)$ & \\
\hline TNM stage & & & 0.156 \\
\hline I & $17(11)$ & $372(16)$ & \\
\hline II & $70(43)$ & $924(41)$ & \\
\hline III-IV & $74(46)$ & $964(43)$ & \\
\hline Histologic grade & & & 0.379 \\
\hline I & $32(20)$ & 413 (18) & \\
\hline II & $88(54)$ & $1398(62)$ & \\
\hline III & $42(26)$ & $454(20)$ & \\
\hline ER & & & $<0.001$ \\
\hline- & $52(32)$ & $318(15)$ & \\
\hline+ & $111(68)$ & $1818(85)$ & \\
\hline PR & & & $<0.001$ \\
\hline- & $59(36)$ & $473(22)$ & \\
\hline+ & $104(64)$ & $1658(78)$ & \\
\hline HER-2 & & & 0.390 \\
\hline $0 / 1+$ & $96(60)$ & $1341(63)$ & \\
\hline $2+$ & $53(33)$ & 606 (29) & \\
\hline $3+$ & $11(7)$ & $184(9)$ & \\
\hline LVI & & & 0.030 \\
\hline- & $76(47)$ & $1746(55)$ & \\
\hline+ & $87(53)$ & $530(45)$ & \\
\hline LNM with ECE & & & 0.163 \\
\hline- & $103(63)$ & $1507(68)$ & \\
\hline+ & $60(37)$ & $694(32)$ & \\
\hline $\begin{array}{l}\text { Local regional } \\
\text { recurrence }\end{array}$ & $20(12)$ & $56(5)$ & $<0.001$ \\
\hline Distant metastasis & $68(42)$ & $96(9)$ & $<0.001$ \\
\hline Died of disease & $76(47)$ & $89(8)$ & $<0.001$ \\
\hline Surgery type & & & 0.001 \\
\hline
\end{tabular}

Table 1 (continued)

\begin{tabular}{llll}
\hline Characteristics & $\begin{array}{l}\text { CgIMPC }(n= \\
163)(n, \%)\end{array}$ & $\begin{array}{l}\text { SgIMPC }(n= \\
\text { 2299) }(n, \%)\end{array}$ & $P$ value \\
\hline $\begin{array}{c}\text { Breast-conserving } \\
\text { surgery/Lumpectomy } \\
\quad \text { Mastectomy }\end{array}$ & $5(3)$ & $16(1)$ & \\
$\begin{array}{l}\text { Chemotherapy } \\
\text { (adjuvant/ neoadjuvant) }\end{array}$ & $158(97)$ & $2283(99)$ & \\
0 & $13(8)$ & $153(7)$ & \\
1 & $150(92)$ & $2146(93)$ & \\
Radiation therapy & & $1060(46)$ & \\
0 & $69(42)$ & $1239(54)$ & \\
1 & $94(58)$ & $308(15)$ & \\
Endocrine therapy & & $1794(85)$ & \\
0 & $72(47)$ & & \\
1 & $80(53)$ & & \\
\hline
\end{tabular}

Cg IMPC control group of invasive micropapillary carcinoma, $S g I M P C$ study group of invasive micropapillary carcinoma, $L N M$ lymph node metastasis, LVI lymphovascular invasion, ER estrogen receptor, $P R$ progesterone receptor, HER-2 human epidermal growth factor receptor 2, ECE extracapsular extension

receptor ( 85 vs. $68 \% ; p<0.001)$ and progesterone-receptor expression (78 vs.64\%; $p<0.001$ ), lower rate of locoregional recurrence ( 5 vs. $12 \% ; p<0.001)$ and distant metastasis ( 9 vs. $42 \% ; p<0.001$ ), and fewer breast cancer specific death $(8$ vs. $47 \% ; p<0.001)$. In addition, the study group was noted to occur in older patients $(p<0.001)$. Histologic grade, human epidermal growth factor receptor 2, and lymph node stage were not significantly different between the study group and control group $(p>0.05)$.

The proportion of luminal type carcinoma by immunohistochemistry was $87 \%$ in the study group, significantly higher than that in the control group $(75 \% ; p<0.001)$.

\section{Treatment}

The surgical and adjuvant treatments of breast cancer patients were summarized in Table 1. A total of 2283 (99\%) patients in the study group and $158(97 \%)$ patients in the control group underwent total mastectomy or modified radical mastectomy. The remaining patients in each group were treated with breast-conserving surgery. Compared with the patients in the study group, fewer patients in the control group underwent mastectomy $(p=0.001)$. Fewer patients in the control group received endocrine therapy $(p<0.001)$, while a similar proportion of patients in the two groups received adjuvant chemotherapy and radiation therapy. 
Fig. 2 Disease-free survival (a) and overall survival (b) of patients in the study group and control group. Patient in the study group had a more favorable disease-free survival and overall survival than that in the control group
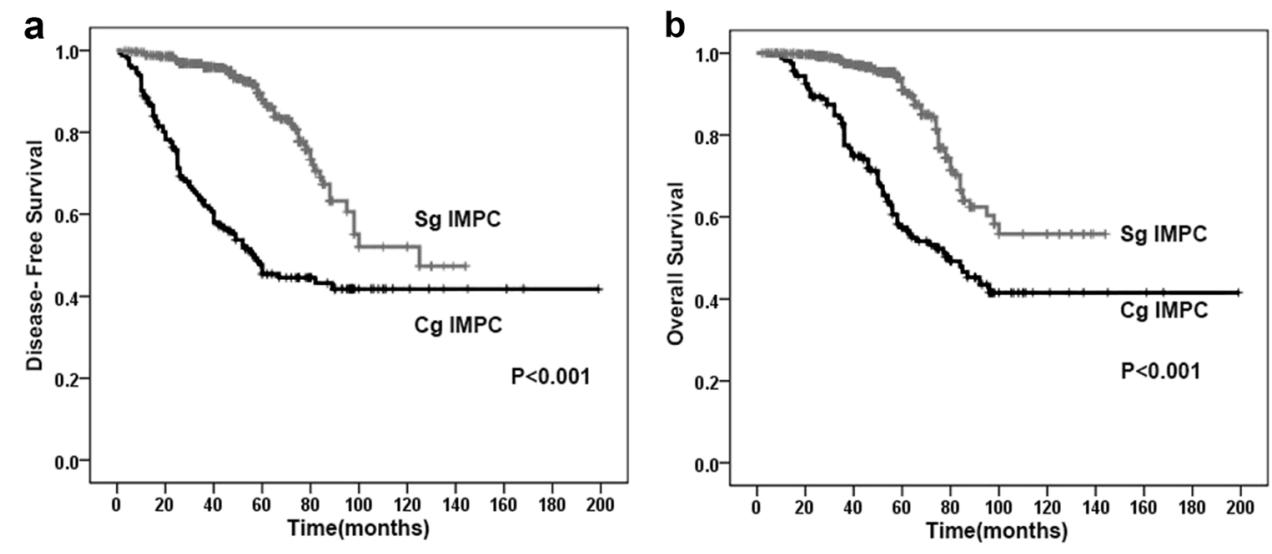

\section{Survival comparison}

The median follow-up was 54 months (range 3-144 months) in the study group and 56 months (range 4-199 months) in the control group. The 5-year disease-free survival and overall survival for patients in the study group were 83.8 and $90.9 \%$, respectively, compared with 45.4 and $57.4 \%$ in the control group (Fig. 2). Patient in the study group had a better disease-free survival and overall survival. The 10-year disease-free survival for patients in the study group and control group were 47.3 and $41.8 \%$, respectively. The 10 -year overall survival for patients in the study group and control group were 55.8 and $48.0 \%$, respectively. There was no significant difference in disease-free survival and overall survival among patients with different percentage of invasive micropapillary carcinoma component in the study group or in the control group (Fig. 3).

Univariate analysis showed that tumor size, lymphovascular invasion, lymph node metastasis, estrogen receptor and progesterone receptor status were significant prognostic factors in the control group $(p<0.05)$ (Table 2). In addition to tumor size, lymphovascular invasion, lymph node metastasis, estrogen receptor and progesterone receptor status, histologic grade and human epidermal growth factor receptor 2 were also significant prognostic factors in the study group $(p<0.05)$ (Table 3$)$. Multivariate analysis using Cox regression showed that lymphovascular invasion and estrogen receptor status were significant prognostic factors in the control group $(p<0.05)$ (Table 2). Lymphovascular invasion and estrogen receptor were the most significant prognostic factor, followed by lymph node metastasis and tumor size in the study group $(p<0.05)$ (Table 3). The proportion of invasive micropapillary carcinoma and histologic grade did not significantly correlate with survival in either invasive micropapillary carcinoma group.

\section{Discussion}

Invasive micropapillary carcinoma is a special histologic subtype of breast cancer with aggressive behavior. Our group conducted a series of studies on invasive micropapillary carcinoma of the breast in recent years and the results provided a basis for the diagnosis and treatment of invasive micropapillary carcinoma patients [6, 13, 19-27].

It is well known that breast cancer may have mixed histologic patterns within the same tumor. Some histologic subtypes have different clinical behaviors. Precise pathological diagnosis therefore is critical for clinical management of the tumor with mixed histology. Marchio et al. [31] compare pure invasive micropapillary carcinomas with the grade- and hormone-receptor status-matched invasive ductal carcinomas using genomic analysis. They demonstrated that invasive micropapillary carcinomas had distinct molecular genetic profiles. Furthermore, the authors [32] also compared 24 pure and 40 mixed invasive micropapillary carcinomas and reported that mixed invasive micropapillary carcinomas are more closely related to pure invasive micropapillary carcinoma than to invasive ductal carcinoma. Thus, micropapillary morphology in breast cancer constitutes a distinct entity at both histologic and genetic levels, regardless of the proportions of the invasive micropapillary carcinoma component.

Our current and previous studies [6, 13, 19] demonstrated that, even if a small proportion of invasive micropapillary carcinoma was present in a tumor, the rate of lymph node metastasis and the number of lymph node metastasis per case were significantly increased. As previously reported [6], current study indicated that the proportion of invasive micropapillary carcinoma did not significantly correlate with survival in the two invasive micropapillary carcinoma groups. Therefore, any invasive micropapillary carcinoma component should be recognized to guide the individualized treatment, and the extent of invasive micropapillary carcinoma component should be 
Fig. 3 Kaplan-Meier estimates disease-free survival and overall survival, comparing the four categories of different proportions of invasive micropapillary carcinoma in the study group $(\mathbf{a}, \mathbf{b})$ and control group $(\mathbf{c}, \mathbf{d})$. There was no significant difference in diseasefree survival and overall survival, among the different proportions of invasive micropapillary carcinoma in the study group and control group
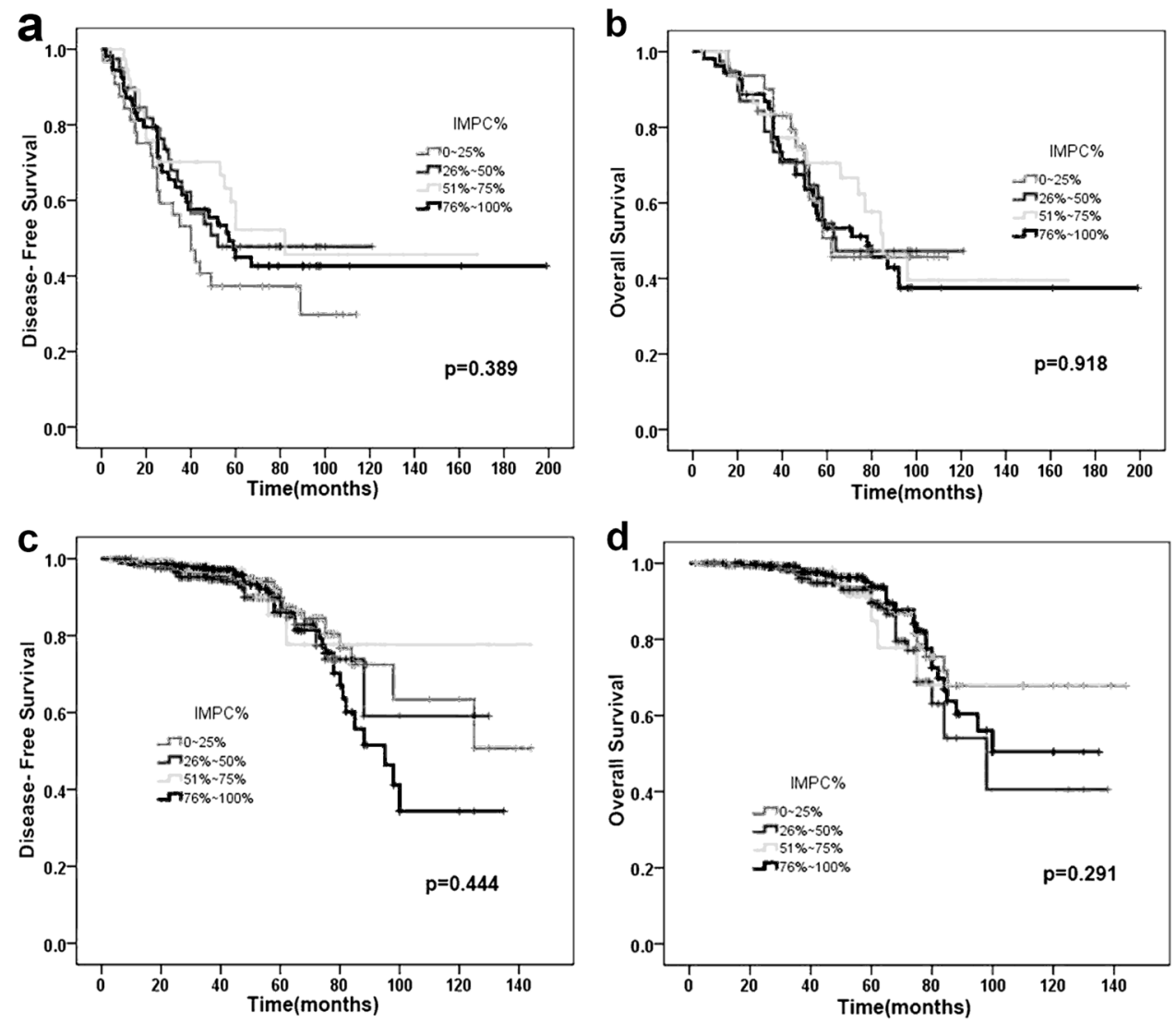

indicated in the pathology report. In a tumor with mixed histology, the lack of reporting the percentage of invasive micropapillary carcinoma component might have contributed to the different incidence rates of invasive micropapillary carcinoma in the literature. In addition to the variation in diagnostic criteria, incomplete specimen sampling of the tumor is another contributing factor.

In our study, we examined the entire tumor and breast tissue, and the diagnosis of invasive micropapillary carcinoma was further verified by immunohistochemical staining. Invasive micropapillary carcinoma component was identified in $6 \%$ of the cases in the study group, significantly higher than that that in the control group $(2 \%)$. The diagnostic rate of invasive micropapillary carcinoma in recent years was increased annually and reached $7 \%$ in 2015. Increased awareness of this uncommon but aggressive tumor component plays a pivotal role in our accurate diagnosis.

Our previous study [6] indicated that invasive micropapillary carcinoma did not respond well to adjuvant chemotherapy with Cyclophosphamide Methotrexate Fluorouracil, but endocrine therapy improved patient outcomes. As noted in Table 1, the majority of the study group were positive for estrogen receptor and/or progesterone receptor. Therefore, patients in this group were not treated with Cyclophosphamide Methotrexate Fluorouracil chemotherapy; instead, those with estrogen receptor and progesterone receptor expression were given hormone therapy (oral Tamoxifen or Aromatase inhibitors) or ovarian oblation. When invasive micropapillary carcinoma component was identified and indicated in the pathology report, the patients would be followed-up in shorter intervals, with more frequent imaging surveillance, and a drug sensitivity test was conducted in patients with recurrence or metastasis. With the modification of therapeutic regimen based upon the precise pathologic diagnosis, we found that the outcomes of patients in the study group were significantly better than those in the retrospective control group with a lower rate of metastasis and mortality. Our findings indicate that as long as invasive micropapillary carcinoma is accurately diagnosed and reported, the prognosis of invasive micropapillary carcinoma can be improved by appropriate treatment tailored to the individual patient. With precise diagnosis and individualized treatments, the 5-year diseasefree survival and overall survival of patients in the study group were improved to 83.8 and $90.9 \%$, respectively, compared to 45.4 and $57.4 \%$ in the control group.

Lymphovascular invasion and lymph node metastasis were unfavorable prognostic indicators for breast cancer. Our study showed that lymphovascular invasion and ER were the most significant prognostic factors in both study group and control group in multivariate analyses. Lymph 
Table 2 Univariate and multivariate analyses of clinicopathological features for prognosis of control group invasive micropapillary carcinoma

\begin{tabular}{|c|c|c|c|c|c|c|}
\hline \multirow[t]{2}{*}{ Factor } & \multicolumn{3}{|c|}{ Univariate } & \multicolumn{3}{|c|}{ Multivariate } \\
\hline & HR & $95 \% \mathrm{CI}$ & $p$ & HR & $95 \% \mathrm{CI}$ & $p$ \\
\hline \multicolumn{7}{|l|}{ DFS } \\
\hline Tumor size & 1.671 & $1.124-2.483$ & 0.011 & 1.417 & $0.937-2.144$ & 0.099 \\
\hline LNM & 3.073 & $1.416-6.667$ & 0.005 & 1.776 & $0.732-4.307$ & 0.204 \\
\hline LVI & 9.110 & $5.067-16.379$ & $<0.001$ & 7.969 & $4.208-15.094$ & $<0.001$ \\
\hline Histologic grade & 1.164 & $0.843-1.606$ & 0.367 & - & - & - \\
\hline ER & 0.331 & $0.215-0.510$ & $<0.001$ & 0.506 & $0.260-0.984$ & 0.045 \\
\hline PR & 0.374 & $0.244-0.574$ & $<0.001$ & 0.769 & $0.397-1.488$ & 0.435 \\
\hline HER-2 & 1.143 & $0.891-1.466$ & 0.294 & - & - & - \\
\hline IMPC composition & 0.912 & $0.755-1.103$ & 0.342 & - & - & - \\
\hline \multicolumn{7}{|l|}{ OS } \\
\hline Tumor size & 1.633 & $1.083-2.464$ & 0.019 & 1.209 & $0.770-1.898$ & 0.409 \\
\hline LNM & 3.112 & $1.350-7.174$ & 0.008 & 1.486 & $0.600-3.680$ & 0.392 \\
\hline LVI & 8.781 & $4.688-16.447$ & $<0.001$ & 8.330 & $4.059-17.098$ & $<0.001$ \\
\hline Histologic grade & 1.379 & $0.975-1.949$ & 0.069 & - & - & - \\
\hline ER & 0.289 & $0.184-0.455$ & $<0.001$ & 0.420 & $0.209-0.844$ & 0.015 \\
\hline PR & 0.330 & $0.210-0.520$ & $<0.001$ & 0.768 & $0.391-1.506$ & 0.442 \\
\hline HER-2 & 1.123 & $0.869-1.452$ & 0.376 & - & - & - \\
\hline IMPC composition & 1.033 & $0.843-1.266$ & 0.755 & - & - & - \\
\hline
\end{tabular}

$H R$ hazard ratio, 95\% CI 95\% confidence interval, LNM lymph node metastasis, LVI lymphovascular invasion, ER estrogen receptor, $P R$ progesterone receptor, HER-2 human epidermal growth factor receptor 2, DFS disease-free survival, OS overall survival

Table 3 Univariate and multivariate analyses of clinicopathological features for prognosis of study group invasive micropapillary carcinoma

\begin{tabular}{|c|c|c|c|c|c|c|}
\hline \multirow[t]{2}{*}{ Factor } & \multicolumn{3}{|c|}{ Univariate } & \multicolumn{3}{|c|}{ Multivariate } \\
\hline & HR & $95 \% \mathrm{CI}$ & $p$ value & HR & $95 \% \mathrm{CI}$ & $p$ value \\
\hline \multicolumn{7}{|l|}{ DFS } \\
\hline Tumor size & 2.573 & $1.797-3.684$ & $<0.001$ & 1.665 & $1.122-2.470$ & 0.011 \\
\hline LNM & 5.692 & $2.630-12.318$ & $<0.001$ & 3.224 & $1.125-8.593$ & 0.018 \\
\hline LVI & 5.264 & $3.217-8.614$ & $<0.001$ & 2.737 & $1.531-4.895$ & 0.001 \\
\hline Histologic grade & 2.231 & $1.623-3.066$ & $<0.001$ & 1.183 & $0.818-1.712$ & 0.372 \\
\hline ER & 0.235 & $0.156-0.353$ & $<0.001$ & 0.299 & $0.174-0.516$ & $<0.001$ \\
\hline PR & 0.405 & $0.269-0.609$ & $<0.001$ & 0.907 & $0.538-1.531$ & 0.715 \\
\hline HER-2 & 1.248 & $1.031-1.511$ & 0.021 & 1.023 & $0.836-1.251$ & 0.826 \\
\hline IMPC composition & 1.084 & $0.931-1.264$ & 0.299 & - & - & - \\
\hline \multicolumn{7}{|l|}{ OS } \\
\hline Tumor size & 2.384 & $1.618-3.511$ & $<0.001$ & 1.443 & $0.933-2.199$ & 0.100 \\
\hline LNM & 8.112 & $2.973-22.140$ & $<0.001$ & 6.219 & $1.456-26.563$ & 0.014 \\
\hline LVI & 5.005 & $2.909-8.611$ & $<0.001$ & 2.688 & $1.420-5.090$ & 0.002 \\
\hline Histologic grade & 2.121 & $1.506-2.988$ & $<0.001$ & 1.219 & $0.813-1.828$ & 0.338 \\
\hline ER & 0.286 & $0.184-0.445$ & $<0.001$ & 0.448 & $0.246-0.816$ & 0.009 \\
\hline PR & 0.422 & $0.272-0.656$ & $<0.001$ & 0.773 & $0.428-1.397$ & 0.394 \\
\hline HER-2 & 1.274 & $1.041-1.559$ & 0.019 & 1.071 & $0.869-1.321$ & 0.519 \\
\hline IMPC composition & 1.006 & $0.853-1.186$ & 0.942 & - & - & - \\
\hline
\end{tabular}

HR hazard ratio, 95\% CI 95\% confidence interval, LNM lymph node metastasis, LVI lymphovascular invasion, ER estrogen receptor, PR progesterone receptor, HER-2 human epidermal growth factor receptor 2, DFS disease-free survival, $O S$ overall survival 
node metastasis and tumor size were also significant prognostic factors in the study group. It is hence important to accurately assess lymphovascular invasion [15] and hormone receptor status [33].

\section{Conclusions}

In the era of the control group, some invasive micropapillary carcinomas were not recognized due to the lack of awareness, and more importantly, the method of wholespecimen orientation and serial sectioning was not implemented. Lack of close follow-up and adjuvant endocrine therapy in these patients seemed to have led to a higher rate of local recurrence and distant metastasis. All cases in the study group were examined using the method of wholespecimen orientation and serial sectioning. Our data suggest that these patients survivals were improved significantly because of the precise pathological diagnosis and individualized treatment.

In summary, invasive micropapillary carcinoma is a special type of breast cancer with aggressive behaviors. Even if invasive micropapillary carcinoma is present as a minor component in the tumor, it appears to dominate the clinical and biological behaviors of the tumor. Precise pathological diagnosis and individualized treatment can improve the outcomes of patients with invasive micropapillary carcinoma.

Acknowledgements This study was funded by National Natural Science Foundation of China (30470667, 30600225, 30930038), Program for Chang Jiang Scholars and Innovative Research Team in University of Ministry of Education of China (IRT0743). Hannah Y Wen was supported in part by a National Institutes of Health/National Cancer Institute Cancer Center Support Grant (P30CA008748).

\section{Compliance with Ethical Standards}

Ethical statement All procedures performed in studies involving human (the specimen of patients) participants were in accordance with the ethical standards of the Ethics Committee of the Tianjin Medical Cancer Institute and Hospital.

Conflict of interest The authors declare that they have no conflict of interest.

\section{References}

1. Fisher ER, Palekar AS, Redmond C, et al. Pathologic findings from the National Surgical Adjuvant Breast Project (protocol no. 4). VI. Invasive papillary cancer. Am J Clin Pathol. 1980;73:313-22.

2. Siriaunkgul S, Tavassoli FA. Invasive micropapillary carcinoma of the breast. Mod Pathol. 1993;6:660-2.

3. Amin MB, Ro JY, el-Sharkawy T, et al. Micropapillary variant of transitional cell carcinoma of the urinary bladder. Histologic pattern resembling ovarian papillary serous carcinoma. Am J Surg Pathol. 1994; 18:1224-32.

4. Sakamoto K, Watanabe M, De La Cruz C, et al. Primary invasive micropapillary carcinoma of the colon. Histopathology. 2005;47:479-84.

5. Kuroda N, Hamaguchi N, Ohara M, et al. Intracytoplasmic lumina in invasive micropapillary carcinoma of the lung. Diagn Cytopathol. 2006;34:224-6.

6. Chen L, Fan Y, Lang RG, et al. Breast carcinoma with micropapillary features: clinicopathologic study and long-term followup of 100 cases. Int J Surg Pathol. 2008;16:155-63.

7. Acs G, Esposito NN, Rakosy Z, et al. Invasive ductal carcinomas of the breast showing partial reversed cell polarity are associated with lymphatic tumor spread and may represent part of a spectrum of invasive micropapillary carcinoma. Am J Surg Pathol. 2010;34:1637-46.

8. Chen AC, Paulino AC, Schwartz MR, et al. Prognostic markers for invasive micropapillary carcinoma of the breast: a populationbased analysis. Clin Breast Cancer. 2013;13:133-9.

9. Chen AC, Paulino AC, Schwartz MR, et al. Population-based comparison of prognostic factors in invasive micropapillary and invasive ductal carcinoma of the breast. $\mathrm{Br} \mathrm{J}$ Cancer. 2014;111:619-22.

10. Liu Y, Huang X, Bi R, et al. Similar prognoses for invasive micropapillary breast carcinoma and pure invasive ductal carcinoma: a retrospectively matched cohort study in China. PLoS ONE. 2014;9:e106564.

11. Shi WB, Yang LJ, Hu X, et al. Clinico-pathological features and prognosis of invasive micropapillary carcinoma compared to invasive ductal carcinoma: a population-based study from China. PLoS ONE. 2014;9:e101390.

12. Yu JI, Choi DH, Huh SJ, et al. Differences in prognostic factors and failure patterns between invasive micropapillary carcinoma and carcinoma with micropapillary component versus invasive ductal carcinoma of the breast: retrospective multicenter casecontrol study (KROG 13-06). Clin Breast Cancer. 2015;15:353-61. e351-352

13. Fu L, Ikuo M, Fu XY, et al. Relationship between biologic behavior and morphologic features of invasive micropapillary carcinoma of the breast. Zhonghua Bing Li Xue Za Zhi. 2004;33:21-25.

14. Nassar $\mathrm{H}$, Wallis $\mathrm{T}$, Andea A, et al. Clinicopathologic analysis of invasive micropapillary differentiation in breast carcinoma. Mod Pathol. 2001;14:836-41.

15. Yang YL, Liu BB, Zhang X, et al. Invasive Micropapillary Carcinoma of the Breast: An Update. Arch Pathol Lab Med. 2016;140:799-805.

16. Zekioglu O, Erhan Y, Ciris M, et al. Invasive micropapillary carcinoma of the breast: high incidence of lymph node metastasis with extranodal extension and its immunohistochemical profile compared with invasive ductal carcinoma. Histopathology. 2004:44:18-23.

17. Tavassoli FA, Devilee P. Tumours of the breast and female genital organs.WHO classification of tumours. Pathol Genet. Lyon: IARC; 2003. p. 10.

18. Lakhani SR, Ellis O, Schnitt SJ, et al. WHO classification of tumours of the breast. Lyon: IARC, 2012. p. 8.

19. Guo X, Fan Y, Lang R, et al. Tumor infiltrating lymphocytes differ in invasive micropapillary carcinoma and medullary carcinoma of breast. Mod Pathol. 2008;21:1101-7.

20. Cui LF, Guo XJ, Wei J, et al. Overexpression of TNF-alpha and TNFRII in invasive micropapillary carcinoma of the breast: clinicopathological correlations. Histopathology . 2008;53:381-8.

21. Wei J, Cui L, Liu F, et al. E-selectin and Sialyl Lewis X expression is associated with lymph node metastasis of invasive 
micropapillary carcinoma of the breast. Int $\mathrm{J}$ Surg Pathol. 2010;18:193-200.

22. Liu F, Lang R, Wei J, et al. Increased expression of SDF-1/ CXCR4 is associated with lymph node metastasis of invasive micropapillary carcinoma of the breast. Histopathology. 2009;54:741-50.

23. Li W, Yang D, Wang S, et al. Increased expression of CD146 and microvessel density (MVD) in invasive micropapillary carcinoma of the breast: Comparative study with invasive ductal carcinomanot otherwise specified. Pathol Res Pract. 2011;207:739-46.

24. Li W, Liu F, Lei $\mathrm{T}$, et al. The clinicopathological significance of CD44 + /CD24-/low and CD24 + tumor cells in invasive micropapillary carcinoma of the breast. Pathol Res Pract. 2010;206:828-34.

25. Chen L, Zhu Z, Sun X, et al. Down-regulation of tumor suppressor gene FEZ1/LZTS1 in breast carcinoma involves promoter methylation and associates with metastasis. Breast Cancer Res Treat. 2009;116:471-8.

26. Li S, Yang C, Zhai L, et al. Deep sequencing reveals small RNA characterization of invasive micropapillary carcinomas of the breast. Breast Cancer Res Treat. 2012;136:77-87.

27. Wang XX, Liu BB, Wu X, et al. Loss of leucine zipper putative tumor suppressor 1 (LZTS1) expression contributes to lymph node metastasis of breast invasive micropapillary carcinoma. Pathol Oncol Res. 2015;21:1021-6.
28. Fu L, Tschiya S, Matsyama I, et al. Clinicopathologic features and incidence of invasive lobular carcinoma in Japanese women. Pathol Inter. 1998;48:348-54.

29. Hammond ME, Hayes DF, Dowsett M, et al. American Society of Clinical Oncology/College Of American Pathologists guideline recommendations for immunohistochemical testing of estrogen and progesterone receptors in breast cancer. J Clin Oncol. 2010;28:2784-95.

30. Wolff AC, Hammond ME, Hicks DG, et al. Recommendations for human epidermal growth factor receptor 2 testing in breast cancer: American Society of Clinical Oncology/College of American Pathologists clinical practice guideline update. J Clin Oncol. 2013;31:3997-4013.

31. Marchio C, Iravani M, Natrajan R, et al. Genomic and immunophenotypical characterization of pure micropapillary carcinomas of the breast. J Pathol. 2008;215:398-410.

32. Marchio C, Iravani M, Natrajan R, et al. Mixed micropapillaryductal carcinomas of the breast: a genomic and immunohistochemical analysis of morphologically distinct components. J Pathol. 2009;218:301-15.

33. Luna-More $\mathrm{S}$, Casquero S, Perez-Mellado A, et al. Importance of estrogen receptors for the behavior of invasive micropapillary carcinoma of the breast. Review of 68 cases with follow-up of 54 . Pathol Res Pract. 2000;196:35-39.

\section{Affiliations}

\section{Weidong $\mathrm{Li}^{1,2} \cdot$ Yunwei Han ${ }^{1,2} \cdot$ Chijuan Wang ${ }^{1,2} \cdot$ Xiaojing Guo ${ }^{1,2} \cdot$ Beibei Shen ${ }^{1,2} \cdot$ Fangfang Liu ${ }^{1,2} \cdot$ Chengying Jiang ${ }^{1,2} \cdot$ Yaqing $\mathrm{Li}^{1,2} \cdot$ Yiling Yang ${ }^{1,2} \cdot$ Ronggang Lang $^{1,2} \cdot \mathrm{Yu} \mathrm{Fan}^{1,2} \cdot \mathrm{Feng} \mathrm{Gu}^{1,2} \cdot \mathrm{Yun} \mathrm{Niu}^{1,2}$. Xinmin Zhang ${ }^{3} \cdot$ Lanjing Zhang $^{4,5,6,7} \cdot$ Hannah Y Wen ${ }^{8} \cdot$ Li Fu $^{1,2}$}

1 Department of Breast Cancer Pathology and Research Laboratory, Tianjin Medical University Cancer Institute and Hospital; National Clinical Research Center of Cancer; Key Laboratory of Breast Cancer Prevention and Therapy, Tianjin Medical University, Ministry of Education; Key Laboratory of Cancer Prevention and Therapy; State Key Laboratory of Breast Cancer Research, Tianjin, China

2011 Collaborative Innovation Center of Tianjin for Medical Epigenetics, Tianjin, China

3 Department of Pathology, Cooper University Hospital, Cooper Medical School of Rowan University, One Cooper Plaza, Camden, NJ, USA
4 Department of Pathology, University Medical Center of Princeton, Plainsboro, NJ, USA

5 Department of Biological Sciences, Rutgers University, Newark, NJ, USA

6 Rutgers Cancer Institute of New Jersey, New Brunswick, NJ, USA

7 Department of Chemical Biology, Ernest Mario School of Pharmacy Rutgers University, Piscataway, NJ, USA

8 Department of Pathology, Memorial Sloan Kettering Cancer Center, 1275 York Avenue, New York, NY, USA 